\title{
Rapid Characterization of Hydrogen Evolution Electrocatalyst Spot Arrays by the Pulsed- Potential Substrate Generation/Tip Collection Mode of Scanning Electrochemical Microscopy
}

\author{
Minshu Du* \\ Department of Materials Science and Engineering \\ China University of Petroleum \\ Beijing, China \\ Center for Electrochemistry, Department of Chemistry \\ The University of Texas at Austin \\ Austin, United States
}

dms1223@126.com

\begin{abstract}
A rapid method for the characterization of hydrogen evolution catalysts by scanning electrochemical microscopy (SECM) with decreased $\mathrm{H}_{2}$ diffusion layer overlap is introduced. The double-pulsed substrate generation/tip collection (SG/TC) mode was employed by switching the substrate electrode potential between an inactive potential and an HER active potential while the tip electrode was moved to each catalyst spot and collected the $\mathrm{H}_{2}$. The optimal pulse potential and pulse width were selected using 3D COMSOL simulation. The reliability of this method was confirmed by the good agreement with the results obtained by the traditional SECM screening techniques.
\end{abstract}

Keywords-scanning electrochemical microscopy; energy materials; HER catalysts; substrate generation-tip collection Mode.

\section{INTRODUCTION}

Scanning electrochemical microscopy $[1,2]$ (SECM) has been developed as a powerful technique to study the mechanisms and kinetics of the electrochemical reactions [3]. Both the feedback mode [4] and generation/collection modes [5, 6] offer significant advantages for the quantitative detection of reaction intermediates and measurements of the electrontransfer kinetics. Electrocatalyst screening techniques based on SECM show incomparable versatility in the comparison and selection of electrocatalysts and photoelectrocatalysts [5, 7-10]. In order to probe more catalytic spots during a screening experiment, the distance between spots should be controlled as small as possible. However, a problem arises here is the overlap of diffusion profiles of neighboring spots in the array $[11,12]$. A problem has been solved by developing a tipshielding layer $(\mathrm{Au})$ outside the traditional SECM tip in order to consume diffused products coming from the neighbor spots in the array [13]. However, this method is time-consuming and requires preparing the particular tip, thus could not be extended

M. Du is grateful for the support of the China Scholarship Council (CSC) and Microstructure Laboratory for Energy Materials of China University of Petroleum, Beijing. to other research systems. In addition, Minguzzi's group also raised a method for decreasing the oxygen diffusion layer overlap by reducing the reaction time of OER catalyst spots [14]. However, this method is difficult to operate because the tip has to be approached to each spot in order to test each spot's activity.

In this paper, we applied the similar method based on the pulsed-potential substrate generation-tip collection (SG-TC) mode of SECM for the characterization of Au HER arrays and compared the results to the traditional screening techniques. The optimal double-pulse potential profile applied to the substrate was established by digital simulation using COMSOL Multiphysics software. This method is highly efficient and could be applied to other electrocatalysts and photoelectrocatalysts characterizations. Also it's easy to be operated, and the tip is just needed to be approached to the substrate for only one time. Moreover, we obtained excellent agreement between this new methodology and the traditional SECM screening results, thus allowing a direct comparison and verifying the benefit of our approach.

\section{EXPERIMENTAL SECTION}

\section{A. Chemicals}

$\mathrm{HAuCl}_{4} \cdot 3 \mathrm{H}_{2} \mathrm{O}$ 99.99\% (Alfa Aesar, Ward Hill, MA) was used as received. For piezo-dispensing, the concentration of $\mathrm{Au}$ precursor solution was $0.10 \mathrm{M}$ in ethylene glycol (Fisher, Fair Lawn, NJ). Ferrocenemethanol 97\% (Aldrich, St. Louis, MO), $\mathrm{KNO}_{3}$ (Fisher, Fair Lawn, NJ) and $\mathrm{H}_{2} \mathrm{SO}_{4}$ (Fisher, Fair Lawn, $\mathrm{NJ})$ were used to prepare the aqueous solutions with Milli-Q water $(18.2 \Omega \cdot \mathrm{cm})$.

\section{B. Tip Electrode Preparation}

A $2 \mathrm{~cm}$ long $25 \mu \mathrm{m}$ diameter Pt wire (Alfa Aesar, 99.95\% purity, Ward Hill, MA) was sealed into a borosilicate capillary 
with $1.5 \mathrm{~mm}$ outer diameter and $0.75 \mathrm{~mm}$ inner diameter (FHC, Bowdoin, ME) under vacuum. One end was polished with 600 mesh sandpaper until the metal disk was exposed and then sharpened and smoothed with a $3 \mu \mathrm{m}$ diamond lapping film and a $0.1 \mu \mathrm{m}$ diamond lapping film (Allied, Rancho Dominguez, CA) successively. The RG of the tip (ratio of glass to metal radius) was about 2. Silver-epoxy cured overnight at $100{ }^{\circ} \mathrm{C}$ was used to make electric contact between the Pt wire and a $0.143 \mathrm{~mm}$ diameter $\mathrm{NiCr}$ wire (Alfa Aesar, Ward Hill, MA).

\section{Au Spot Arrays Preparation}

Au arrays were prepared on FTO substrate by the reduction of metal salt precursor using the method described in the previous paper. In brief, $0.1 \mathrm{M} \mathrm{HAuCl}_{4} \cdot 3 \mathrm{H}_{2} \mathrm{O}$ was dissolved in the $0.45 \mu \mathrm{m}$-filtered ethylene glycol solvent. Au arrays were dispensed using a piezo-dispenser (CHI 1550, CH Instruments, Austin, TX). Then the plate was dried under Ar at $120^{\circ} \mathrm{C}$ for 1 $\mathrm{h}$ and reduced under the $5 \% \mathrm{H}_{2}$ and $95 \% \mathrm{Ar}$ mixture atmosphere at $350{ }^{\circ} \mathrm{C}$ for $2 \mathrm{~h}$ in a tube furnace.

\section{Electrochemical Measurements}

A 920C SECM (CH Instruments, Austin, TX) set-up was employed for the electrochemical measurements. A $25-\mu \mathrm{m}$ diameter Pt UME served as the tip electrode, a Pt foil was used as a counter electrode, and a $\mathrm{Ag} / \mathrm{AgCl}(1 \mathrm{M})$ electrode acted as a reference electrode. All the potentials showed in this paper were relative to $\mathrm{Ag} / \mathrm{AgCl}(1 \mathrm{M})$ reference electrode. The pulsed-potential SG-TC experiment and the SECM screening measurement were performed in $50 \mathrm{mM} \mathrm{H}_{2} \mathrm{SO}_{4}$ solution after deaeration (Ar bubbling).

\section{E. Digital Simulation}

3D Digital simulations were performed on COMSOL Multiphysics Software v4.2 (COMSOL, Inc., Burlington, MA). $\mathrm{H}_{2}$ concentration was determined by Fick's Second Law of Diffusion:

$$
\frac{\partial C_{0}}{\partial_{t}}=D_{n} \nabla^{2} C_{n}
$$

The flux boundary condition was set as shown in (2):

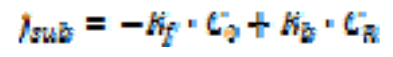

The time-dependent substrate current was calculated using Fick's First Law of Diffusion:

$$
\frac{i}{r F A}=-T(t)=D_{D} F C_{D}
$$

\section{RESUlTS AND DISCUSSION}

Three rows of $\mathrm{Au}$ catalyst arrays were dispended on the FTO substrate, as shown in Fig. 1. Each row was dispensed 5, 10 and 15 drops of precursor solution (100 pL per drop) from upper to the lower, respectively. The shapes of prepared spots were not the perfect circle as expected because of the unavoidable tilt of the substrate during thermal-reduction processes in the tube furnace. It is noticed that certain spots were not uniform, e.g., the lower left spot that was indicated as $2^{\#}$ included one area with darker color as highlighted as $5^{\#}$. This nonuniformity might be caused by the limited dissolving capacity of $0.1 \mathrm{M} \mathrm{HAuCl}_{4} \cdot 3 \mathrm{H}_{2} \mathrm{O}$ in the ethylene glycol solvent.

The optimal pulse width was determined by digital simulation using COMSOL Multiphysics Software. A scheme of the simulation geometry and the governing equations was reported in Fig. 2. The hydrogen flux $J$ that diffused away from Au spots caused the time-dependent growth of a diffusion layer which was decided by the boundary conditions showed in (2). Varying the pulsed width from $1.5 \mathrm{~s}$ to $300 \mathrm{~s}$, different $\mathrm{H}_{2}$ concentration distributions in the XY plane were expressed by color-coded profiles. In Fig. 3, the spot with larger HER rate constant $k^{0}$ showed higher $\mathrm{H}_{2}$ concentration, which agreed with the actual activity. And the diffusion profiles in Fig. $3 \mathrm{c}$ and $3 \mathrm{~d}$, where the spots were kept active for the longer time of $100 \mathrm{~s}$ and $300 \mathrm{~s}$, overlapped significantly and interfere with an accurate determination of $\mathrm{H}_{2}$ profile above the addressed spot. In contrast, pulse potential for only $1.5 \mathrm{~s}$ as it done in Fig. 3a yield a well-resolved diffusion profile that reflected each spot's activity. As a result, the pulsed width was chosen as $1.0 \mathrm{~s}$ in this paper.

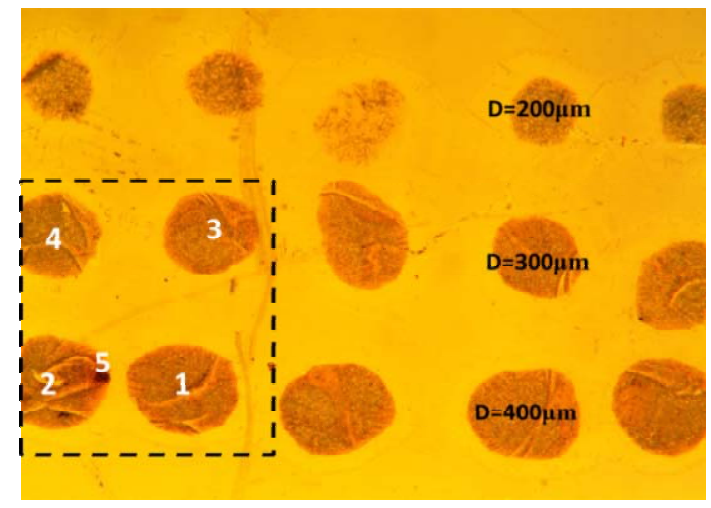

Fig. 1. Optical microscope image of dispensed Au catalyst arrays. Specific spots or positions as highlighted by $1^{\#}$ to $5^{\#}$ are the chosen microelectrodes for SECM.

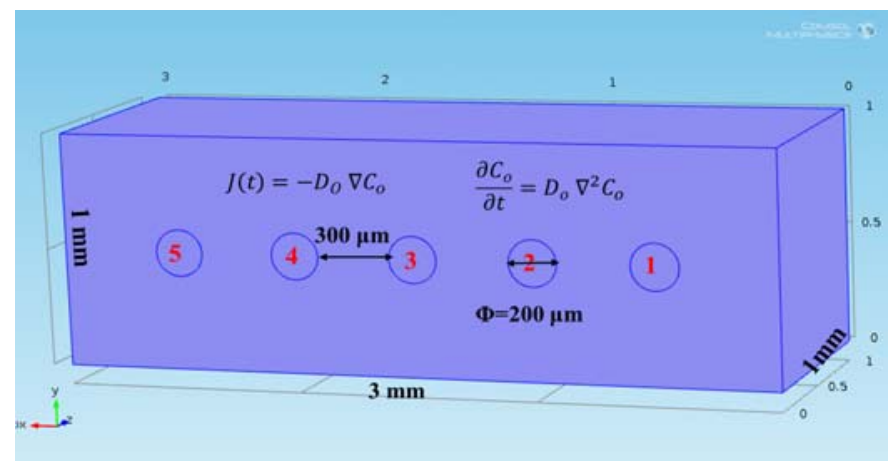

Fig. 2. Schematic design of geometry and governing equations relevant to the 3D ( $\mathrm{x}, \mathrm{y}, \mathrm{z}$ space) digital simulations used in the COMSOL software. HER is treated as a simple one-electron transfer reduction reaction, $J$ indicates the diffusive flux of $\mathrm{H}_{2}$ and Do is the diffusion coefficient of the proton. 

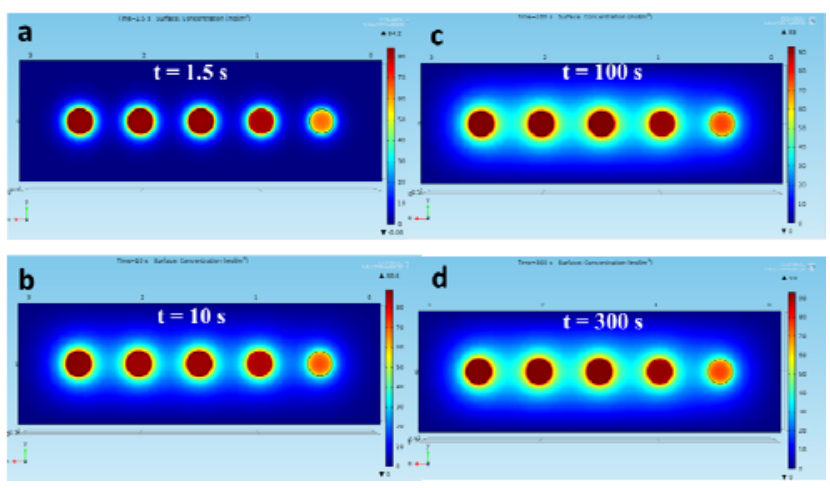

Fig. 3. Simulated color-coded profiles of $\mathrm{H}_{2}$ concentration in the $\mathrm{XY}$ plane when Au spots were biased at $-0.7 \mathrm{~V}$ for the different time. 5 spots represent different HER activities by given the rate constant $\left(k^{0}\right)$ to be $0.001,0.01,0.1$, 1.0 and $10 \mathrm{~cm} / \mathrm{s}$ from right to left, respectively.

Before the SG-TC experiments, Pt tip was approached to the $\mathrm{Au}$ arrays substrate using a standard redox mediator of 1 $\mathrm{mM}$ ferrocenemethanol in $0.1 \mathrm{M} \mathrm{KNO}_{3}$. The Pt tip was biased at $0.4 \mathrm{~V}$ to oxidize the ferrocenemethanol $(\mathrm{FcMeOH})$ under steady state while the substrate potential was biased at $0 \mathrm{~V}$ for ferrocene reduction. As the tip approached the substrate in the $\mathrm{Z}$ direction, there was a typical positive feedback curve (showed in Fig. 4). According to the empirical equation in the reference ${ }^{5}$, the distance between tip and substrate was predicted to be $3.8 \mu \mathrm{m}$. And the fitting curve in red was in good agreement with the experimental approach curve in black in Fig. 4.

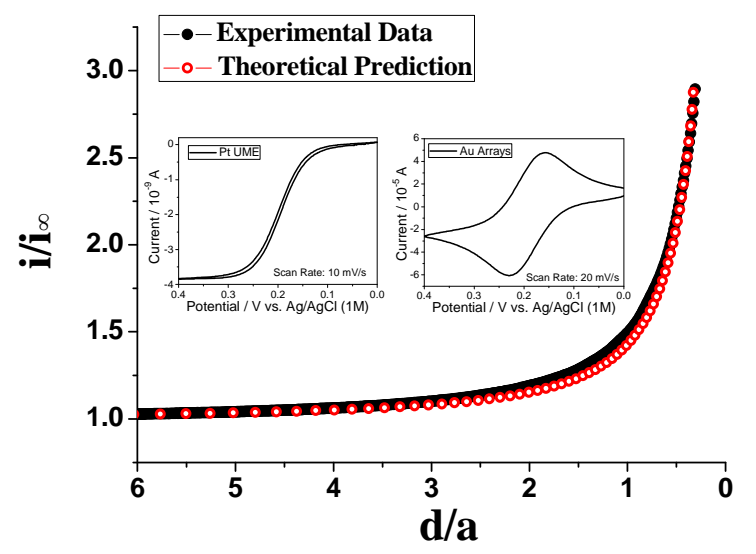

Fig. 4. SECM approach curve upon Au arrays in $1 \mathrm{mM}$ ferrocenemethanol based on the positive feedback mode. The Y axial is the normalized tip current and the $\mathrm{X}$ axial is the normalized distance that is equal to $d / a$, where $\mathrm{d}$ is the real tip-substrate distance and $a$ is the radius of the tip. The insets are the cyclic voltammograms of $\mathrm{Pt}$ tip and Au arrays in $1 \mathrm{mM}$ ferrocenemethanol solution with $0.1 \mathrm{M} \mathrm{KNO}_{3}$ as supporting electrolyte.

Pulsed-potential SG-TC mode was employed by generating $\mathrm{H} 2$ at substrate through HER and collecting $\mathrm{H} 2$ on tip through hydrogen oxidation reaction (HOR). Since the tip current was proportional to the $\mathrm{H} 2$ concentration, as shown in Fig. 5, it showed a clear oscillating behavior between the background current (about 0 A) and HOR current when the substrate potential was switched between the open circuit potential
(OCP) and a negative potential of $-0.7 \mathrm{~V}$ for HER in $50 \mathrm{mM}$ H2SO4 solution. Obviously, spot 5 displayed the highest activity and spot 3, 4 displayed the lowest activity, which was caused by the differences of Au particle amounts in each spot.

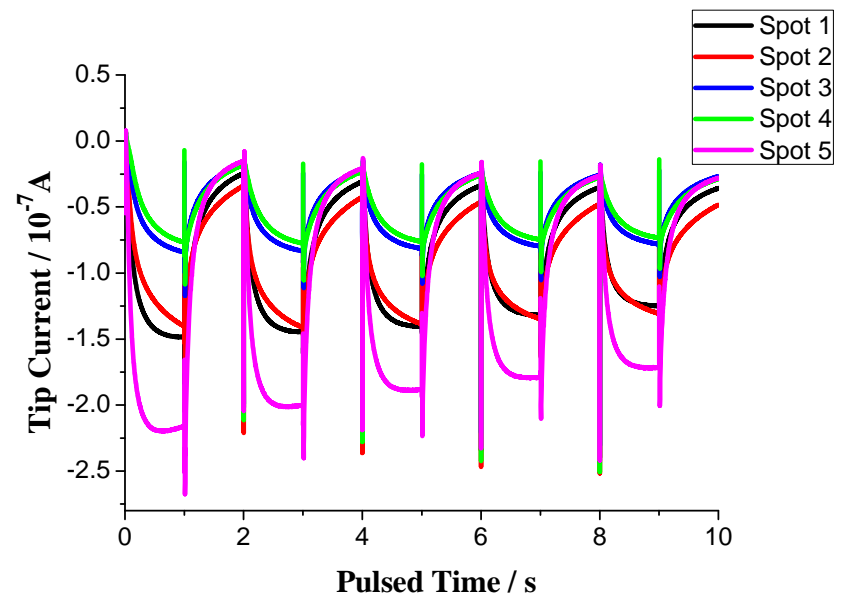

Fig. 5. Oscillated tip current according to the double-pulse potential applied to the substrate at 5 specific positions in $50 \mathrm{mM} \mathrm{H}_{2} \mathrm{SO}_{4}$. Tip potential was held at $0 \mathrm{~V}$, and the substrate potential was switched between open circuit potential $(\mathrm{OCP})$ and $-0.7 \mathrm{~V}$. The pulse width is $1.0 \mathrm{~s}$ with 5 replicates.
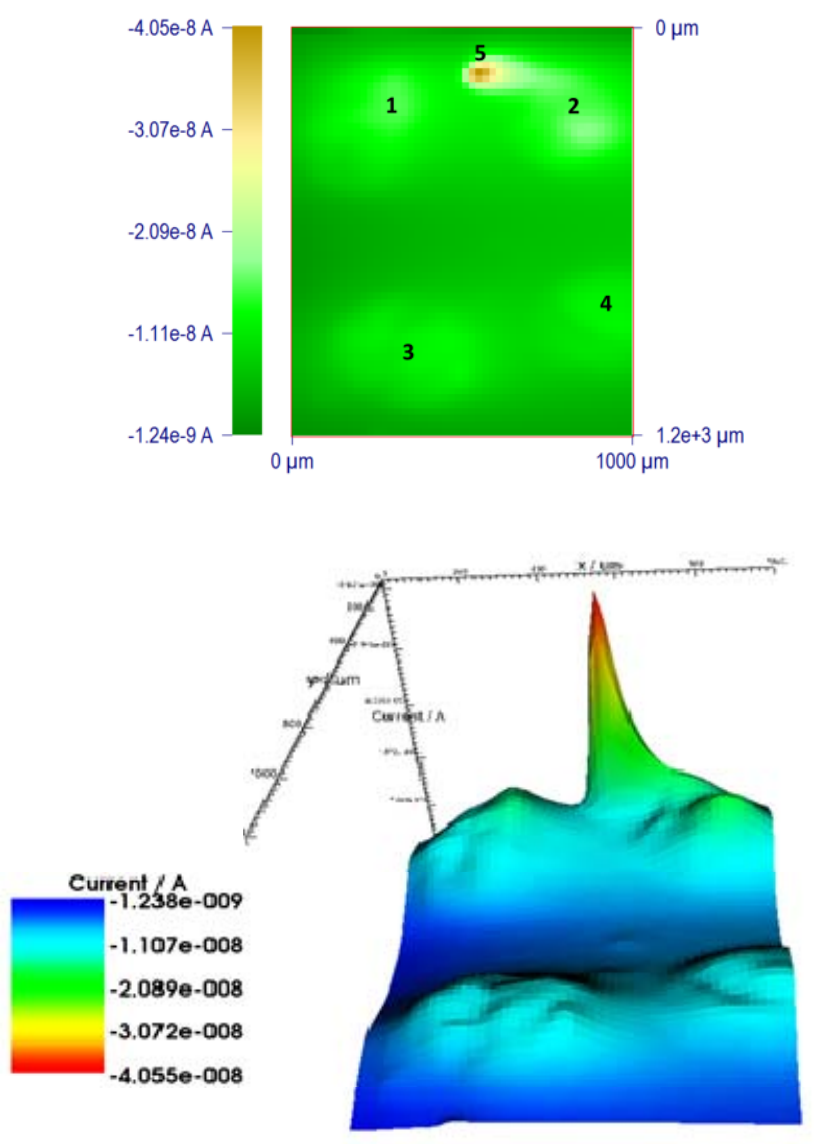

Fig. 6. 2D (upper) and interactive 3D (lower) images of Au arrays by the SECM screening experiment. Color bar indicates the tip collection current that is proportional to the activities of Au catalysts. 
At last, SECM screening experiment was done, in which the Pt tip was held at $0 \mathrm{~V}$ and scanned in the $\mathrm{XY}$ plane at a scan rate of $100 \mu \mathrm{m}$ per $0.2 \mathrm{~s}$. From Fig. 6, both 2D and 3D images indicated the spots HER activities ranked as: $5>1,2>$ 3 , 4 , which agreed with the conclusion obtained from pulsedpotential SG-TC experiment.

\section{CONCLUSION}

In this paper, we introduced the pulsed-potential SG-TC mode of SECM for the characterization of HER catalyst arrays and compared the results to the SECM screening technique. It is an improvement to the traditional SG-TC mode of SECM that solves the partial overlap of diffusion profile of neighboring spots in the arrays. By switching the substrate potential between the inactive potential (OCP in this paper) and active potential, the overlap of diffusion layer could be suppressed by selecting an appropriate pulse time. We also provided a method for choosing the optical pulse potential and pulse width by 3D COMSOL simulation. Moreover, the timeconsuming adjustment of substrate tilting in the SECM screening technique could be simplified, and the problem of gas bubble forming in the OER or HER could be avoided in terms of relatively short reaction time in this pulsed-potential SG-TC mode. The electrocatalytic activity ranking obtained from pulsed-potential SG-TC mode agreed well with the SECM screening results, which verified the reliability of this repaid characterization method.

In addition, it is believed that this method will be extended to the characterization of other electrocatalysts and photoelectrocatalysts. It displays the superiority when the substrate is intrinsically non-flat or bent and when the catalyst arrays are prepared with short distance between each other.

\section{REFERENCES}

[1] A.J. Bard, F.R.F. Fan, J. Kwak, and O. Lev, "Scanning electrochemical microscopy. introduction and principles," Anal. Chem., vol. 61, pp. 132138, 1989.

[2] A.J. Bard and M.V. Mirkin, Mirkin, Scanning electrochemical microscopy, CRC Press, 2012

[3] P.R. Unwin and A.J. Bard, "Scanning electrochemical microscopy. 9. Theory and appliction of the feedback mode to the measurement of following chemical reaction rates in electrode process," J. Phys. Chem., vol. 95, pp. 7814-7824, 1991.

[4] A.J. Bard, M.V. Mirkin, P.R. Unwin and D.O. Wipf, "Scanning electrochemical microscopy. 12. Theory and experiment of the feedback mode with finite heterogeneous electron-transfer kinetics and arbitrary substrate size,” J. Phys. Chem., vol. 96, pp. 1861-1868, 1992.

[5] C.M. Sánchez-Sánchez, J. Rodríguez-López and A.J. Bard, "Scanning electrochemical microscopy. 60. Quantitative calibration of the SECM substrate generation/tip collection mode and its use for the study of the oxygen reduction mechanism," Anal. Chem., vol. 80, pp. 3254-3260, 2008.

[6] J.L. Fernandez and A.J. Bard, "Scanning electrochemical microscopy. 47. Imaging electrocatalytic activity for oxygen reduction in an acidic medium by the tip generation-substrate collection mode," Anal. Chem. Vol. 75, pp. 2967-2974, 2003.

[7] J.L. Fernández, D.A. Walsh and A.J. Bard, "Thermodynamic guidelines for the design of bimetallic catalysts for oxygen electroreduction and rapid screening by scanning electrochemical microscopy. M-Co (M: Pd, $\mathrm{Ag}, \mathrm{Au})$," J. Amer. Chem. Soc., vol. 127, pp. 357-365, 2005.

[8] C. Jung, C.M. Sánchez-Sánchez, C.L. Lin, J. n. Rodríguez-López and A.J. Bard, "Electrocatalytic activity of Pd-Co bimetallic mixtures for formic acid oxidation studied by scanning electrochemical microscopy," Anal. Chem., vol. 81, pp. 7003-7008, 2009.

[9] Y.C. Weng and C.T. Hsieh, "Scanning electrochemical microscopy characterization of bimetallic $\mathrm{Pt}-\mathrm{M}(\mathrm{M}=\mathrm{Pd}, \mathrm{Ru}, \mathrm{Ir})$ catalysts for hydrogen oxidation," Electrochim. Acta, vol. 56, pp. 1932-1940, 2011.

[10] C. Bhattacharya, H.C. Lee and A.J. Bard, "Rapid screening by scanning electrochemical microscopy (SECM) of dopants for $\mathrm{Bi}_{2} \mathrm{WO}_{6}$ improved photocatalytic water oxidation with Zn doping," J. Phys. Chem. C, vol. 117, pp. 9633-9640, 2013.

[11] O. Sklyar, M. Träuble, C. Zhao and G. Wittstock, "Modeling steadystate experiments with a scanning electrochemical microscope involving several independent diffusing species using the boundary element method," J. Phys. Chem. B, vol. 110, pp. 15869-15877, 2006.

[12] K. Eckhard, X.X. Chen, F. Turcu and W. Schuhmann, "Redox competition mode of scanning electrochemical microscopy (RC-SECM) for visualisation of local catalytic activity", Phys. Chem. Chem. Phys. vol. 8, pp. 5359-5365, 2006.

[13] A. Minguzzi, M.A. Alpuche-aviles, J.R. lopez, S. Rondinini and A.J. Bard, "Scanning of oxygen evolution electrocatalysts by scanning electrochemical microscopy using a shielded tip approach", Anal. Chem. vol. 80, pp. 4055-4064, 2008.

[14] A. Minguzzi, D. Battistel, J. Rodríguez-López, A. Vertova, S. Rondinini, A. J. Bard and S. Daniele, "Rapid Characterization of Oxygen-Evolving Electrocatalyst Spot Arrays by the Substrate Generation/Tip Collection Mode of Scanning Electrochemical Microscopy with Decreased $\mathrm{O}_{2}$ Diffusion Layer Overlap,” J. Phys. Chem. C, vol. 119, pp. 2941-2947, 2015 . 